\title{
An approximate quantum Hamiltonian identification algorithm using a Taylor expansion of the matrix exponential function *
}

\author{
Yuanlong Wang ${ }^{1,2}$, Daoyi Dong ${ }^{1}$ and Ian R. Petersen ${ }^{3}$
}

\begin{abstract}
An approximate quantum Hamiltonian identification algorithm is presented with the assumption that the system initial state and observation matrix can be set appropriately. We sample the system with a fixed period and using the sampled data we estimate the Hamiltonian based on a Taylor expansion of the matrix exponential function. We prove the estimation error is linear in the variance of the additive Gaussian noise. We also propose a heuristic formula to find the order of magnitude of the optimal sampling period. Two numerical examples are presented to validate the theoretical results on robustness-analysis.
\end{abstract}

\section{INTRODUCTION}

Quantum tomography has been receiving increasing attention as it provides a way to characterize an unknown quantum object [1-6]. When the object is a quantum process, the task is called quantum process tomography [1]. Standard quantum process tomography methods are designed for general quantum process. It is possible to design more efficient algorithms for specific quantum processes. The system Hamiltonian can be taken as an important special class of quantum processes that are of primary interest for quantum systems [2]. The task of identifying parameters in the system Hamiltonian is called Quantum Hamiltonian Identification (QHI) or Hamiltonian Tomography.

Some QHI methods have already been designed for special classes of Hamiltonians. For example, Sone and Cappellaro [9] employed the Gröbner basis to investigate the Hamiltonian identifiability of a many-body spin system assisted by measuring a single quantum probe. Based on quantum process tomography, Wang et al. [7] designed a general iterative identification method for time-independent Hamiltonians, which is then improved in [8] as a two-step optimization QHI method. Bonnabel et al. [10] proposed a symmetry-preserving observer-based quantum Hamiltonian identification and proved an exponential convergence result for 2-level systems with the atom-laser frequency detuning and coupling constant being the unknown parameters. Bris et al. [11] investigated the problem of identifying the field free Hamiltonian and/or the dipole moment of a

*This work was supported by the Australian Research Council's Discovery Projects funding scheme under Project DP130101658 and Laureate Fellowship FL110100020, and the AFOSR under grant FA2386-16-1-4065.

${ }^{1}$ School of Engineering and Information Technology, University of New South Wales, Canberra, ACT 2600, Australia yuanlong.wang.qcegmail.com, daoyidong@gmail. com

${ }^{2}$ Centre for Quantum Computation and Communication Technology, Australian Research Council, Canberra, ACT 2600, Australia

${ }^{3}$ Research School of Engineering, Australian National University, Canberra, ACT 0200, Australia i.r.petersen@gmail. com quantum system. They proved the well-posedness of the problem and introduced a numerical approach to estimate the parameters. Mohseni et al. [12] presented an approach to directly estimate single- and two-qubit Hamiltonian parameters using a single measurement device. Cole et al. [13] developed a Hamiltonian estimation method for some two-state systems and provided uncertainty bounds on the parameters. Cole et al. [14] also proposed a scheme to characterize 㙂e general Heisenberg Hamiltonian with nonuniform couplings. Holzäpfel et al. [15] designed a scalable method to reconstruct one-dimensional local Hamiltonians, which requires at most linearly many states to be prepared and linearly many observables to be measured. Rudinger and Joynt [16] applied compressed sensing methods to estimate Hamiltonians which are sparse in a well-defined sense. Franco et al. [17] presented a method to determine the coupling parameters in a chain of interacting spins that requires only time-resolved measurements over a single particle and no state initialization. Wang et al. [18] employed dynamical decoupling to determine all the coupling terms in a manybody Hamiltonian with finite energy density, which requires rounds of measurements varying linearly with the coupling term number in the Hamiltonian.

Zhang and Sarovar [19] proposed a QHI method from temporal records of system observables (time traces). They established the QHI problem as a system identification one, which is more familiar to the control systems community. Then they used enenstate realization algorithm (ERA) to construct a system realization from measurement results. By solving polynomial equations obtained from the transfer function, they identified the unknown parameters in the Hamiltonian. Recently, Wang et al. [20] proposed an approximate QHI method based on time traces and a matrix logarithm expansion. Using this method, the truncation error in the final estimation decreases exponentially with the length of experimental data.

In this paper, we follow the idea in [19] and [20] to perform QHI from measurement time traces. Based on a Taylor expansion of the matrix exponential function, we design a new QHI algorithm. We consider the robustness of the proposed algorithm in the presence of Gaussian additive noise, which was not considered in [20]. We prove the that the estimation error in this algorithm is linear in the variance of the noise. We perform numerical simulations to validate the analysis results.

The organization of this paper is as follows. Section II formulates the quantum Hamiltonian identification problem. Section III presents our QHI algorithm, analyzes its ro- 
bustness, and discusses the optimization of the sampling period. Numerical results on the estimation error versus noise variance and sampling period are given in Section IV. Section V concludes this paper and proposes possible directions for future research.

\section{Problem Formulation}

We first rephrase the framework in [19] to formulate the QHI problem. Let $\mathrm{H}$ denote the Hamiltonian to be identified and let $\mathfrak{s u}(d)$ denote the Lie algebra consisting of all $d \times d$ skew-Hermitian matrices. Then $i \mathrm{H} \in \mathfrak{s u}(d)$ where $i=\sqrt{-1}$. Let $\left\{i \mathrm{H}_{j}\right\}\left(j=1,2, \ldots, d^{2}-1\right)$ be a set of orthonormal bases of $\mathfrak{s u}(d)$, with $d \times d$ Hermitian matrices $\mathrm{H}_{\mathrm{j}}$ known and the inner product defined as $\left\langle i \mathrm{H}_{\mathrm{j}}, i \mathrm{H}_{\mathrm{k}}\right\rangle=\operatorname{Tr}\left(\mathrm{H}_{\mathrm{j}}^{\dagger} \mathrm{H}_{\mathrm{k}}\right)$. Then we parameterize the system Hamiltonian $\mathrm{H}$ as

$$
\mathrm{H}=\sum_{m=1}^{M} h_{m} \mathrm{H}_{\mathrm{m}},
$$

where $h_{m} \in \mathcal{R}$ are unknown-parameters to be estimated. $M$ is a known number determined by the specific structure of the consideredquantum system.

Let $C_{j k l}$ denote the structure constants of $\mathfrak{s u}(d)$, which satisfy

$$
\left[i \mathrm{H}_{\mathrm{j}}, i \mathrm{H}_{\mathrm{k}}\right]=\sum_{l=1}^{d^{2}-1} C_{j k l}\left(i \mathrm{H}_{\mathrm{l}}\right), \quad j, k=1, \ldots, d^{2}-1,
$$

where $[A, B]=A B-B A$. Without loss of generality we assume that the $C_{j k l}$ are real. Let $\rho$ be the system state, whose evolution is determined by the Liouville-von Neumann equation

$$
\dot{\rho}=-i[\mathrm{H}, \rho] .
$$

If $\mathrm{H}_{\mathrm{j}}$ is an observable, the experimental data can be obtained from Born's rule

$$
x_{j}=\operatorname{Tr}\left(\mathrm{H}_{\mathrm{j}} \rho\right) .
$$

Combining (1)-(4), we obtain

$$
\dot{x}_{k}=\sum_{l=1}^{d^{2}-1}\left(\sum_{m=1}^{M} C_{m k l} h_{m}\right) x_{l} .
$$

Define a matrix A with its element on $k^{\text {th }}$ row and $l^{\text {th }}$ column as

$$
\mathrm{A}_{k l}=\sum_{m=1}^{M} C_{m k l} h_{m},
$$

and we rewrite (5) in matrix form as

$$
\dot{\mathbf{x}}=\mathrm{Ax}
$$

where $\mathbf{x}=\left(x_{1}, x_{2}, \ldots, x_{d^{2}-1}\right)^{T}$. We can further write the observation equation as

$$
y=\mathbf{c}^{T} \mathbf{x} .
$$

For example, if we only observe $\mathrm{H}_{2}$, then $\mathbf{c}^{T}=$ $(0,1,0, \ldots, 0)$. If we design an algorithm to identify $\mathrm{A}_{k l}$, then we can estimate $h_{m}$ and finally reconstruct $\mathrm{H}$.
Remark 1: To further reduce the dimension of $\mathrm{A}$, one can focus on the Lie subalgebra generated by $\mathrm{H}$ ( $m=$ $1,2, \ldots, M)$. For specific procedures, one can refer to [19] and [21].

It is possible to have $M \ll d^{2}-1$ due to practical requirements (e.g., focusing on several parameters) or physical structures (e.g., spin chain). In such situations, (6) implies that we may only need to estimate some of the parameters in $\mathrm{A}$ to reconstruct $\mathrm{H}$. We define the matrix $\overline{\mathrm{C}}$ as

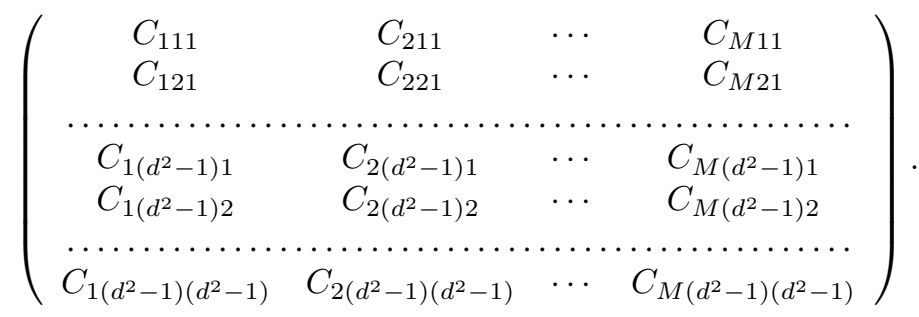

We take $M$ rows from $\overline{\mathrm{C}}$ to form a nonsingular matrix $\tilde{\mathrm{C}}$. For those $\mathrm{A}_{k l}$ whose corresponding $C_{m k l} \in \tilde{\mathrm{C}}$, we write them into an $M \times 1$ vector $\tilde{\mathbf{a}}$. Let

$$
\mathbf{h}=\left(h_{1}, h_{2}, \ldots, h_{M}\right)^{T} \text {. }
$$

Then we have

$$
\tilde{\mathbf{a}}=\tilde{\mathrm{C}} \mathbf{h} .
$$

To estimate $\tilde{\mathbf{a}}$, which may have a much lower dimension than $\mathbf{x}$, we aim to design an efficient algorithm to estimate one element of ã at each time. Let I denote the identity matrix. We formulate the QHI problem as follows:

Problem 1: Consider a real linear system

$$
\left\{\begin{array}{l}
\dot{\mathbf{x}}(t)=\mathrm{Ax}(t), \quad \mathbf{x}(0)=\mathbf{x}_{0}, \\
y(t)=\mathbf{c}^{T} \mathbf{x}(t) .
\end{array}\right.
$$

Let $\mathbf{e}_{\mathbf{j}}$ denote the $j^{\text {th }}$ column of I. We assume that $\mathbf{c}$ and $\mathbf{x}_{0}$ can be chosen as any $\mathbf{e}_{\mathbf{j}}\left(1 \leq j \leq d^{2}-1\right)$ with a proper experimental setup. The QHI problem is to design an efficient method to identify $\mathrm{A}_{a b}\left(1 \leq a, b \leq d^{2}-1\right)$.

\section{HAMILTONIAN IDENTIFICATION AlgORITHM}

\section{A. Identification Algorithm}

In classical control theory, the methods to solve Problem 1 usually take A as a whole and estimate all of its elements [22], and the algorithm efficiency is severely restricted by the dimension of $\mathrm{A}$. In quantum systems, the dimension increases exponentially with the number of least information unit (qubit), which makes many dimension-dependent algorithms impractical for many-qubit systems. Furthermore, when people are only interested in some (instead of all) of the unknown parameters, it will be unnecessary to reconstruct all of the elements in A. Here we propose an effective algorithm on the basis of proper prior knowledge and experimental setting.

Let $\hat{x}$ denote the estimation of 1 variable $x$ in this paper. We assume that the experimental data is obtained through sampling the system with a period $\Delta t$. Suppose $\mathbf{x}_{0}=\mathbf{e}_{\mathbf{k}}$. For the observable $\mathrm{H}_{\mathrm{i}}$, the $j^{\text {th }}$ data value is

$$
\begin{aligned}
\hat{y}(j \Delta t) & =\mathbf{c}^{T} e^{\mathrm{A} j \Delta t} \mathbf{x}_{0} \\
& =\sum_{r=0}^{\infty} \frac{j^{r} \Delta t^{r}}{r !} \mathbf{c}^{T} \mathrm{~A}^{r} \mathbf{x}_{0} \\
& =\delta_{i k}+\sum_{r=1}^{\infty} \frac{j^{r} \Delta t^{r}}{r !}\left(\mathrm{A}^{r}\right)_{i k},
\end{aligned}
$$


where $\delta$ is the Kronecker delta function.

Let $\theta=\left(\theta_{1}, \theta_{2}, \ldots, \theta_{n}\right)^{T}$ where $\theta_{r}=\left(\mathrm{A}^{r}\right)_{i k}$. The determination of the number $n$ will be illustrated later. Suppose the data length is $N$, which is usually determined by experiments. We let

$$
\hat{\mathbf{y}}^{N}=\left[\hat{y}(\Delta t)-\delta_{i k}, \hat{y}(2 \Delta t)-\delta_{i k}, \ldots, \hat{y}(N \Delta t)-\delta_{i k}\right]^{T} .
$$

We truncate the infinite series in (11) and rewrite it in matrix form

$$
\hat{\mathbf{y}}^{N} \approx \mathrm{K} \theta
$$

where

$$
\mathrm{K}=\left(\begin{array}{cccc}
\frac{1^{1} \Delta t^{1}}{1 !} & \frac{1^{2} \Delta t^{2}}{2 !} & \cdots & \frac{1^{n} \Delta t^{n}}{n !} \\
\frac{2^{1} \Delta t^{1}}{1 !} & \frac{2^{2} \Delta t^{2}}{2 !} & \cdots & \frac{2^{n} \Delta t^{n}}{n !} \\
\cdots \ldots & \ldots \ldots \ldots \ldots & \ldots \ldots & \ldots \ldots \ldots \\
\frac{N^{1} \Delta t^{1}}{1 !} & \frac{N^{2} \Delta t^{2}}{2 !} & \cdots & \frac{N^{n} \Delta t^{n}}{n !}
\end{array}\right)_{N \times n} .
$$

Then we use a least squares method to obtain an estimate

$$
\hat{\theta}=\left(K^{T} \mathrm{~K}\right)^{-1} \mathrm{~K}^{T} \hat{\mathbf{y}},
$$

and we have $\hat{\mathrm{A}}_{j k}=\hat{\theta}_{1}$.

The procedure of our algorithm is outlined as follows:

Algorithm 1: Step 1. Determine ã, $\tilde{\mathrm{C}}$ and the sampling period $\Delta t$. $n$ should be chosen such that $n \leq N$.

Step 2. For $C_{m a b} \in \tilde{\mathrm{C}}$, set the initial state as $\mathbf{x}_{0}=\mathbf{e}_{\mathbf{b}}$ and observe $\mathrm{H}_{\mathrm{a}}$. Record the sampled data value $\hat{\mathbf{y}}^{N}$.

Step 3. Estimate $\hat{\theta}$ using (14). Then $\hat{\mathrm{A}}_{a b}=\hat{\theta}_{1}$.

Step 4. Change $a$ and $b$ and repeat Step 2 and Step 3 to estimate all elements in $\tilde{\mathbf{a}}$.

Step 5. $\hat{H}$ is obtained using (1) and (9).

\section{B. Robustness Analysis}

We present the following theorem to characterize the robustness of our algorithm.

Theorem 1: Assume the experimental data is subject to additive Gaussian noise with zero mean and correlation matrix $\sigma^{2} I$. Using our algorithm, the mean squared error (MSE) $E\left[\operatorname{Tr}(\hat{\mathrm{H}}-\mathrm{H})^{2}\right]$ is linear in $\sigma^{2}$, where $E(\cdot)$ denotes expectation.

Proof: Let $\eta$ denote the noise vector such that $\hat{\mathbf{y}}^{N}=$ $\mathbf{y}^{N}+\eta$. Also let

$$
\mathrm{R}=\sum_{r=n+1}^{\infty} \frac{\Delta t^{r}}{r !}\left(\mathrm{A}^{r}\right)_{i k}\left(1^{r}, 2^{r}, \ldots, N^{r}\right)^{T} .
$$

Its convergence can be seen from the fact that the factorial function increases much faster than the power function. The estimated vector is

$$
\begin{aligned}
\hat{\theta} & =\left(\mathrm{K}^{T} \mathrm{~K}\right)^{-1} \mathrm{~K}^{T}\left(\mathrm{y}^{N}+\eta\right) \\
& =\left(\mathrm{K}^{T} \mathrm{~K}\right)^{-1} \mathrm{~K}^{T}(\mathrm{~K} \theta+\mathrm{R}+\eta) \\
& =\theta+\left(\mathrm{K}^{T} \mathrm{~K}\right)^{-1} \mathrm{~K}^{T} \mathrm{R}+\left(\mathrm{K}^{T} \mathrm{~K}\right)^{-1} \mathrm{~K}^{T} \eta .
\end{aligned}
$$

We then have

$$
\begin{aligned}
& E\left[\left(\hat{\theta}_{1}-\theta_{1}\right)^{2}\right] \\
& =\quad E\left[\left(\mathbf{e}_{\mathbf{1}}^{T} \hat{\theta}-\mathbf{e}_{\mathbf{1}}^{T} \theta\right)^{2}\right] \\
& =\mathrm{R}^{T} \mathrm{~K}\left(\mathrm{~K}^{T} \mathrm{~K}\right)^{-1} \mathbf{e}_{\mathbf{1}} \mathbf{e}_{\mathbf{1}}^{T}\left(\mathrm{~K}^{T} \mathrm{~K}\right)^{-1} \mathrm{~K}^{T} \mathrm{R} \\
& \quad+\operatorname{Tr}\left[\mathrm{K}\left(\mathrm{K}^{T} \mathrm{~K}\right)^{-1} \mathbf{e}_{\mathbf{1}} \mathbf{e}_{\mathbf{1}}^{T}\left(\mathrm{~K}^{T} \mathrm{~K}\right)^{-1} \mathrm{~K}^{T} \eta \eta^{T}\right] \\
& =\left[\mathrm{R}^{T} \mathrm{~K}\left(\mathrm{~K}^{T} \mathrm{~K}\right)^{-1} \mathbf{e}_{\mathbf{1}}\right]^{2}+\sigma^{2} \mathbf{e}_{\mathbf{1}}^{T}\left(\mathrm{~K}^{T} \mathrm{~K}\right)^{-1} \mathbf{e}_{\mathbf{1}} .
\end{aligned}
$$

Therefore $E\left[\left(\hat{\mathrm{A}}_{i k}-\mathrm{A}_{i k}\right)^{2}\right]$ is linear in $\sigma^{2}$. Similarly,

$$
E\left[\left(\hat{\mathrm{A}}_{i k}-\mathrm{A}_{i k}\right)\left(\hat{\mathrm{A}}_{a b}-\mathrm{A}_{a b}\right)\right] \quad((i, k) \neq(a, b))
$$

is also linear in $\sigma^{2}$. Furthermore,

$$
\begin{aligned}
& E\left[\operatorname{Tr}(\hat{\mathrm{H}}-\mathrm{H})^{2}\right] \\
& =E\left\{\operatorname{Tr}\left[(\hat{\mathbf{h}}-\mathbf{h})(\hat{\mathbf{h}}-\mathbf{h})^{T}\right]\right\} \\
& \left.\left.=E\left\{\operatorname{Tr}\left[\tilde{\mathbf{C}}^{-T} \tilde{\mathbf{C}}^{-1} \hat{\tilde{\mathbf{a}}}-\tilde{\mathbf{a}}\right) \hat{\hat{\mathbf{a}}}-\tilde{\mathbf{a}}\right)^{T}\right]\right\},
\end{aligned}
$$

which is also linear in $\sigma^{2}$.

\section{Optimization of the Sampling Period}

The sampling period is a key index affecting the final estimation error. We present two factors guiding the choice of $\Delta t$.

First, as illustrated in the supplementary material of [19], the Nyquist Sampling Theorem leads to an upper bound for $\Delta t$. Assume that we have the prior knowledge $\|\mathrm{A}\| \leq F$, where $\|\cdot\|$ is the Frobenius norm. Then we require

$$
\Delta t<\frac{\pi}{F}
$$

Second, (16) leads to a critical value that $\Delta t$ is not recommended to surpass. Let

$$
D_{1}=\mathrm{R}^{T} \mathrm{~K}\left(\mathrm{~K}^{T} \mathrm{~K}\right)^{-1} \mathbf{e}_{\mathbf{1}}
$$

We can say that $D_{1}$ is associated with the norm of R. Using Stirling's Formula, the absolute value of each item in the summation defining $\mathrm{R}$ can be approximated as

$$
\begin{aligned}
& \left|\frac{j^{r} \Delta t^{r}}{r !}\left(\mathrm{A}^{r}\right)_{i k}\right| \\
& \approx \frac{1}{\sqrt{2 \pi r}} \frac{(j \Delta t e)^{r}}{r^{r}}\left|\left(\mathrm{~A}^{r}\right)_{i k}\right| \\
& =\frac{1}{\sqrt{2 \pi r}}\left(\frac{j \Delta t e}{r}\right)^{r}\left|\mathbf{e}_{\mathbf{i}}^{T} \mathrm{~A}^{r} \mathbf{e}_{\mathbf{k}}\right| \\
& \leq \frac{1}{\sqrt{2 \pi r}}\left(\frac{j \Delta t e}{r}\right)^{r}|| \mathbf{e}_{\mathbf{i}}^{T}\|\cdot\| \mathrm{A}\left\|\left.\right|^{r} \cdot\right\| \mathbf{e}_{\mathbf{k}} \| \\
& \leq \frac{1}{\sqrt{2 \pi r}}\left(\frac{N F \Delta t e}{r}\right)^{r} .
\end{aligned}
$$

To add up these terms in (19) from $r=n+1$ to infinity, one can anticipate that if $\frac{N F \Delta t e}{r}$ is less than 1 , then the summation would quickly converge, making this residual error $R$ small enough to reduce the identification error in our algorithm. Therefore it is recommended to have

$$
\frac{N F \Delta t e}{n+1} \leq 1
$$

which indieates implies

$$
\Delta t \leq \frac{n+1}{N F e} .
$$

Remark 2: It is worth mentioning that only the first critical point is strict. The second point is heuristic and relies on proper prior knowledge. It can provide suggestions on choosing the order of magnitude of $\Delta t$. 


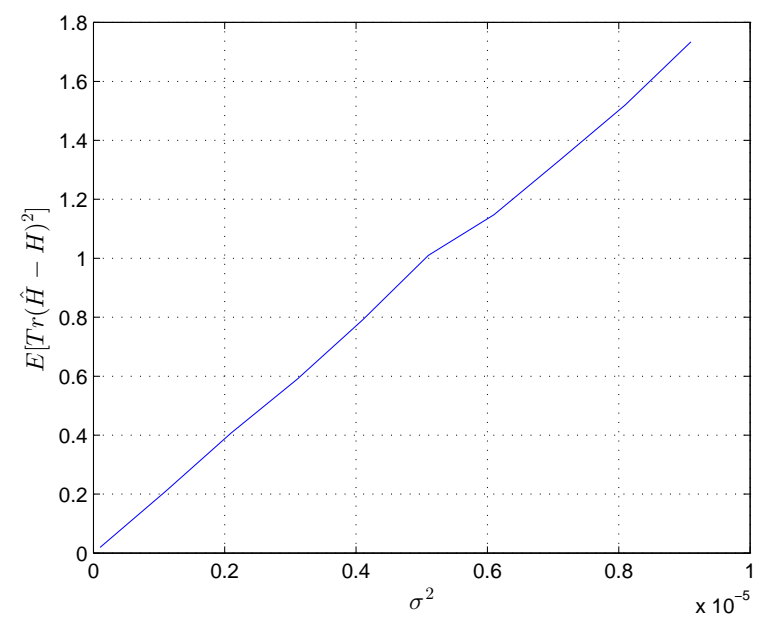

Fig. 1. Identification error $E\left[\operatorname{Tr}(\hat{\mathrm{H}}-\mathrm{H})^{2}\right]$ versus noise variance $\sigma^{2}$ for a single-qubit system.

\section{NumericAl Results}

\section{A. Case Study for a Single-qubit System}

We consider a single-qubit system with Hamiltonian

$$
\mathrm{H}=\sum_{i=x, y, z} f_{i} \sigma_{\mathrm{i}}
$$

where $f_{i}(i=x, y, z)$ are real unknown parameters and the following Pauli matrices are used:

$$
\sigma_{x}=\left(\begin{array}{cc}
0 & 1 \\
1 & 0
\end{array}\right), \quad \sigma_{y}=\left(\begin{array}{cc}
0 & -i \\
i & 0
\end{array}\right), \quad \sigma_{z}=\left(\begin{array}{cc}
1 & 0 \\
0 & -1
\end{array}\right) .
$$

We have

$$
\mathbf{h}=\frac{1}{\sqrt{2}}\left(f_{x}, f_{y}, f_{z}\right)^{T}
$$

and the true value is $(0.1,0.3,-0.8)^{T}$ (units $1 / \mathrm{sec}$ ). The corresponding basis matrices are

$$
\mathrm{H}_{1}=\sigma_{\mathrm{x}}, \mathrm{H}_{2}=\sigma_{\mathrm{y}} \text { and } \mathrm{H}_{3}=\sigma_{\mathrm{z}} \text {. the }
$$

Then the structure constants $C_{j k l}$ are proportional to LeviCivita symbols. We have

$$
\begin{gathered}
\mathrm{A}=\sqrt{2}\left(\begin{array}{ccc}
0 & -f_{3} & f_{2} \\
f_{3} & 0 & -f_{1} \\
-f_{2} & f_{1} & 0
\end{array}\right), \\
\tilde{\mathbf{a}}=\left(\mathrm{A}_{32}, \mathrm{~A}_{13}, \mathrm{~A}_{21}\right)^{T}
\end{gathered}
$$

and

$$
\tilde{\mathrm{C}}=\left(\begin{array}{ccc}
-1 & 0 & 0 \\
0 & -1 & 0 \\
0 & 0 & -1
\end{array}\right)
$$

We first use the proposed algorithm to identify the Hamiltonian under different noise variances. The sampling period used is 0.05 (unit $s$ is omitted henceforth). The data length $N$ is 25 and $h$ is 20 in the simulation. The simulation results shown in Fig. 1, which verifies the conclusion of Theorem 1. Each point is repeated 500 times.

If we make $\sigma=0$, the estimation error will mainly come from the truncation error in our algorithm. We keep

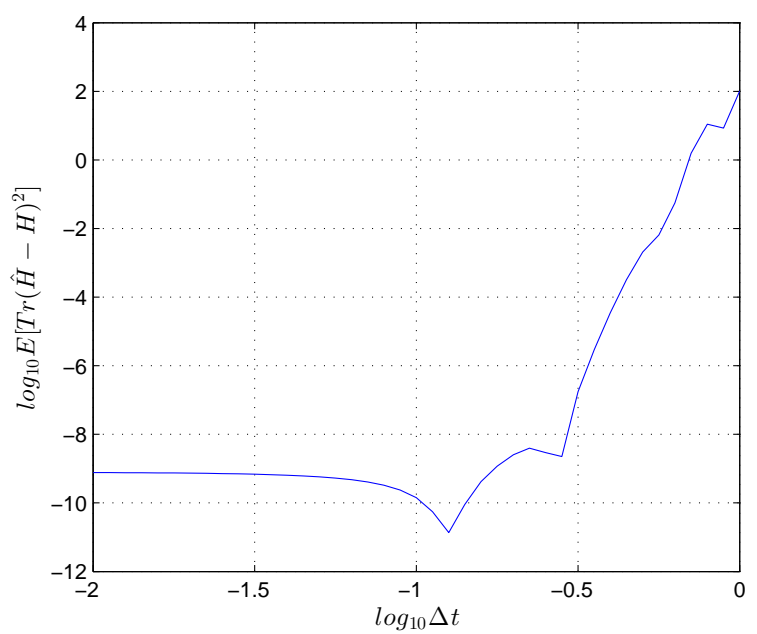

Fig. 2. Truncation error $\log _{10} E\left[\operatorname{Tr}(\hat{\mathrm{H}}-\mathrm{H})^{2}\right]$ versus sampling period $\log _{10} \Delta t$ for a single-qubit system.

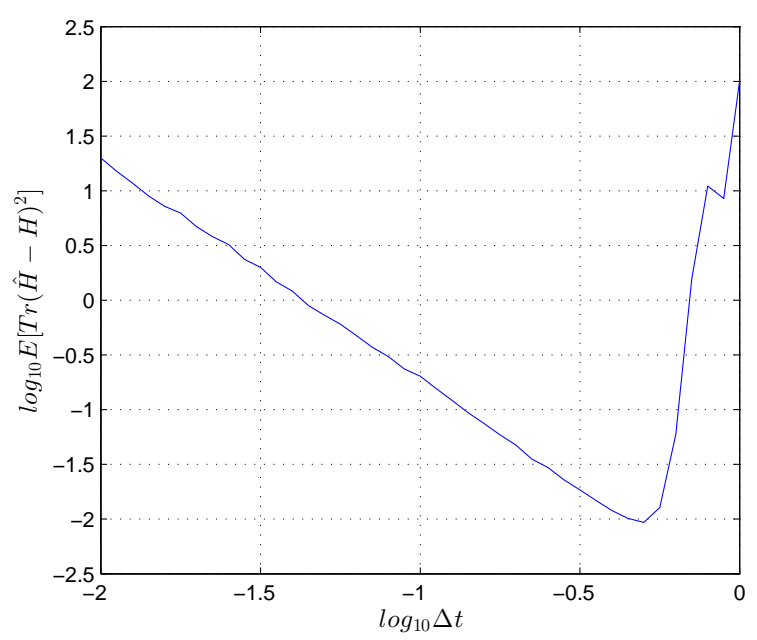

Fig. 3. Identification error $\log _{10} E\left[\operatorname{Tr}(\hat{\mathrm{H}}-\mathrm{H})^{2}\right]$ versus sampling period $\log _{10} \Delta t$ for a single-qubit system.

the sampling period and data length the same as those in Fig. 1. A plot of the truncation error versus sampling period is shown in Fig. 2. As the sampling period increases, the truncation error has an increasing trend, which is in agreement with the analysis in Subsection III-C.

We then simulate the effect of different sampling periods on the estimation error with additive Gaussian noise. We fix the variance of the noise as $\sigma^{2}=4 \times 10^{-6}$ and apply the identification algorithm with different sampling periods. The simulation result is shown in Fig. 3 where each point is repeated 500 times. Assume that we have prior knowledge $\|\mathrm{A}\| \leq 2$. Then (18) gives $\Delta t<2.5824$. Furthermore, (20) gives $\Delta t \leq 0.1472$, which is of the same order of magnitude as the optimal value in Fig. 3. 


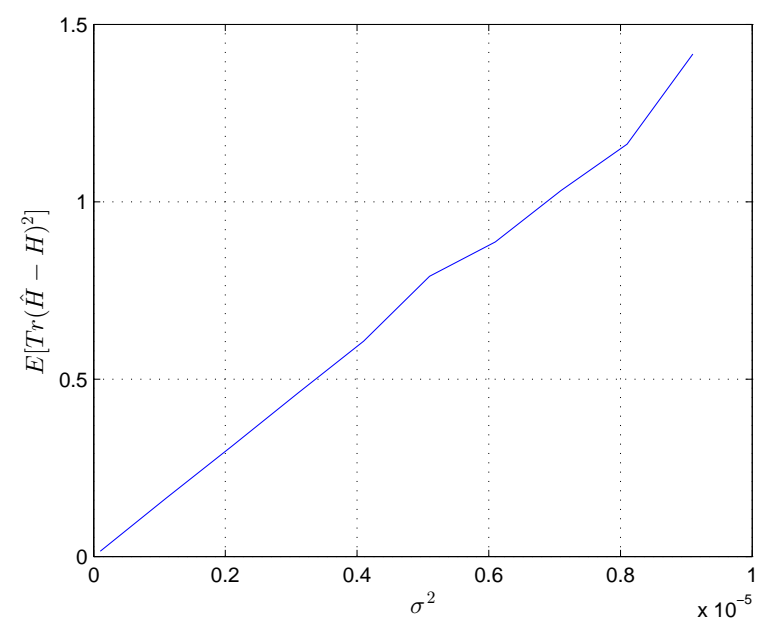

Fig. 4. Identification error $E\left[\operatorname{Tr}(\hat{\mathrm{H}}-\mathrm{H})^{2}\right]$ versus noise variance $\sigma^{2}$ for a 1D Chain System.

\section{B. Case Study for a $1 D$ Chain System}

For multi-qubit systems, we present numerical results for a $1 \mathrm{D}$ chain system with $N_{q}$ qubits:

$$
\mathrm{H}=\sum_{k=1}^{N_{q}} \frac{\omega_{k}}{2} \sigma_{\mathrm{z}}^{\mathrm{k}}+\sum_{k=1}^{N_{q}-1} \delta_{k}\left(\sigma_{+}^{\mathrm{k}} \sigma_{-}^{\mathrm{k}+1}+\sigma_{-}^{\mathrm{k}} \sigma_{+}^{\mathrm{k}+1}\right),
$$

where $\omega_{k}$ and $\delta_{k}$ are the unknown real parameters and $\sigma_{ \pm} \overline{\bar{u}}$ $\sigma_{x} \pm i \sigma_{y}$. This is also the model used in [19]. We focus on $N_{q}=3$. To normalize the basis matrices, we have

$$
\mathbf{h}=\left(\frac{\omega_{1}}{2}, \frac{\omega_{2}}{2}, \frac{\omega_{3}}{2}, 2 \delta_{1}, 2 \delta_{2}\right)^{T}
$$

and the true value is $(3.8,-4,0.4,5.3,-1.1)^{T}$ (units $1 / \mathrm{sec}$ ). The corresponding basis matrices are

$$
\begin{gathered}
\mathrm{H}_{1}=\sigma_{\mathrm{z}}^{1}, \mathrm{H}_{2}=\sigma_{\mathrm{z}}^{2}, \mathrm{H}_{3}=\sigma_{\mathrm{z}}^{3}, \mathrm{H}_{4}=\sigma_{\mathrm{x}}^{1} \sigma_{\mathrm{x}}^{2}, \\
\mathrm{H}_{5}=\sigma_{\mathrm{x}}^{2} \sigma_{\mathrm{x}}^{3}, \mathrm{H}_{6}=\sigma_{\mathrm{y}}^{1} \sigma_{\mathrm{x}}^{2}, \mathrm{H}_{7}=\sigma_{\mathrm{y}}^{2} \sigma_{\mathrm{x}}^{3}, \mathrm{H}_{8}=\sigma_{\mathrm{x}}^{1}, \\
\mathrm{H}_{9}=\sigma_{\mathrm{y}}^{1}, \mathrm{H}_{10}=\sigma_{\mathrm{x}}^{1} \sigma_{\mathrm{y}}^{2}, \mathrm{H}_{11}=\sigma_{\mathrm{x}}^{3}, \mathrm{H}_{12}=\sigma_{\mathrm{y}}^{3} .
\end{gathered}
$$

We then have

$$
\tilde{\mathbf{a}}=\left(\mathrm{A}_{27}, \mathrm{~A}_{16}, \mathrm{~A}_{89}, \mathrm{~A}_{4,10}, \mathrm{~A}_{11,12}\right)^{T}
$$

and

$$
\tilde{\mathrm{C}}=\left(\begin{array}{ccccc}
0 & 0 & 0 & 0 & 2 \\
0 & 0 & 0 & 2 & 0 \\
-2 & 0 & 0 & 0 & 0 \\
0 & -2 & 0 & 0 & 0 \\
0 & 0 & -2 & 0 & 0
\end{array}\right)
$$

We use the proposed algorithm to identify the Hamiltonian under different noise variances. The sampling period we used is 0.05 . The data length $N$ is $25 . n$ is taken as 20 in the simulation. The simulation result is shown in Fig. 4, which is consistent with the conclusion of Theorem 1. Each point is repeated 500 times.

We then set $\sigma=0$ to test the truncation error of our algorithm. We keep the sampling period and data length the same as those in Fig. 4. The result of the truncation error

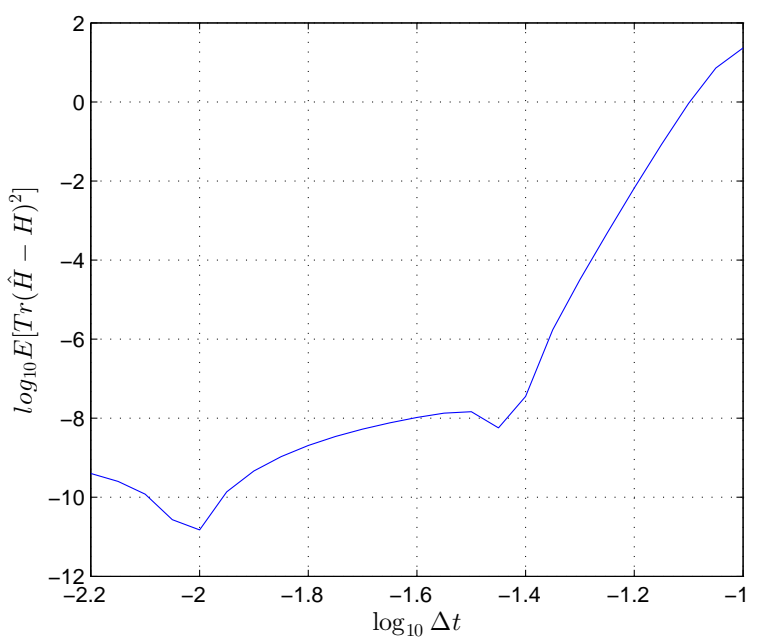

Fig. 5. Truncation error $\log _{10} E\left[\operatorname{Tr}(\hat{\mathrm{H}}-\mathrm{H})^{2}\right]$ versus sampling period $\log _{10} \Delta t$ for a 1D Chain System.

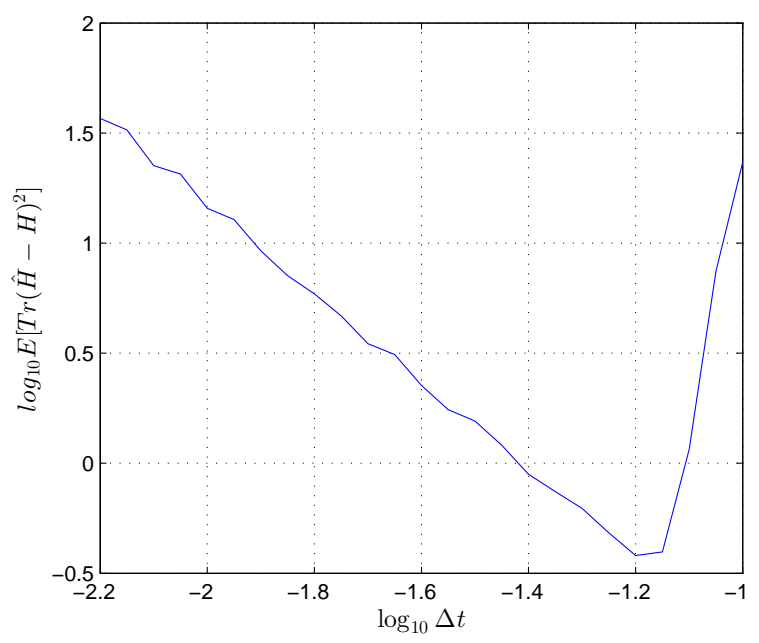

Fig. 6. Identification error $\log _{10} E\left[\operatorname{Tr}(\hat{\mathrm{H}}-\mathrm{H})^{2}\right]$ versus sampling period $\log _{10} \Delta t$ for a 1D Chain System.

versus sampling period is shown in Fig. 5. As the sampling period increases, the truncation error has an increasing trend.

We finally simulate the estimation error with additive Gaussian noise in the measured data under different sampling periods. We fix the variance of the noise as $\sigma^{2}=4 \times$ $10^{-6}$ and apply the identification algorithm under different sampling periods. The numerical result is shown in Fig. 6 and each point is repeated 500 times. Assume that we have prior knowledge $\|\mathrm{A}\| \leq 12$. Then (18) gives $\Delta t<0.5589$. Furthermore, (20) gives $\Delta t \leq 0.0258$, which is of the same order of magnitude as the optimal value in Fig. 6.

\section{CONCLUSION}

We have presented a quantum Hamiltonian identification algorithm under the assumption that the system initial state and observation matrix can be set appropriately. We proved theoreatly that the estimation error is linear in the variance of the additive Gaussian noise and performed simulations to validate this conclusion. We also proposed a heuristic formu- 
la to suggest the order of magnitude of the optimal sampling period. Future work includes a better characterization of the relationship between the estimation error and the sampling period, and possible improvement on the robustness of the algorithm.

\section{REFERENCES}

[1] M. A. Nielsen and I. L. Chuang, Quantum Computation and Quantum Information Cambridge, Cambridge University Press, 2000.

[2] D. Burgarth and K. Yuasa, "Quantum system identification," Phys. Rev. Lett., vol. 108, no. 8, 080502, 2012.

[3] B. Qi, Z. Hou, L. Li, D. Dong, G.-Y. Xiang, G.-C. Guo, "Quantum state tomography via linear regression estimation," Sci. Rep., vol. 3, no. $3496,2013$.

[4] Z. Hou, H.-S. Zhong, Y. Tian, D. Dong, B. Qi, L. Li, Y. Wang, F. Nori, G.-Y. Xiang, C.-F. Li and G.-C. Guo, "Full reconstruction of a 14-qubit state within four hours," New J. Phys., vol. 18, 2016, Art. ID 083036.

[5] B. Qi, Z. Hou, Y. Wang, D. Dong, H.-S. Zhong, L. Li, G.-Y. Xiang, H. M. Wiseman, C.-F. Li and G.-C. Guo, "Adaptive quantum state tomography via linear regression estimation: Theory and two-qubit experiment," npi Quantuln Inform., 3:19, 2017.

[6] M. Guţă and N. Yamamoto, "System identification for passive linear quantum systems," IEEE Trans. Autom. Control, vol. 61, no. 4, pp. 921-936, 2016.

[7] Y. Wang, B. Qi, D. Dong and I. R. Petersen, "An iterative algorithm for Hamiltonian identification of quantum systems," in Proc. 55th IEEE Conf. Decision Control, pp. 2523-2528, Las Vegas, NV, USA, Dec. 12-14, 2016.

[8] Y. Wang, D. Dong, B. Qi, J. Zhang, I. R. Petersen and H. Yonezawa, "A quantum Hamiltonian identification algorithm: computational complexity and error analysis," IEEE Trans. Autom. Control, in press, doi: 10.1109/TAC.2017.2747507, arXiv: 1610.08841, 2016.

[9] A. Sone and P. Cappellaro, "Hamiltonian identifiability assisted by single-probe measurement," Phys. Rev. A, vol. 95, no. 2, 022335, 2017.

[10] S. Bonnabel, M. Mirrahimi and P. Rouchon, "Observer-based Hamiltonian identification for quantum systems," Automatica, vol. 45, no. 5, pp. 1144-1155, 2009

[11] C. Le Bris, M. Mirrahimi, H. Rabitz and G. Turinici, "Hamiltonian identificaiton for quantum systems: well-posedness and numerical approaches," ESAIM: COCV, vol. 13, no. 2, pp. 378-395, 2007.

[12] M. Mohseni, A. T. Rezakhani and A. Aspuru-Guzik, "Direct estimation of single- and two-qubit Hamiltonians and relaxation rates," Phys. Rev. A, vol. 77, no. 4, 042320, 2008.

[13] J. H. Cole, S. G. Schirmer, A. D. Greentree, C. J. Wellard, D. K. L. Oi and L. C. L. Hollenberg, "Identifying an experimental twostate Hamiltonian to arbitrary accuracy," Phys. Rev. A, vol. 71, no. 6 , $062312,2005$.

[14] J. H. Cole, S. J. Devitt and L. C. L. Hollenberg, "Precision characterization of two-qubit Hamiltonians via entanglement mapping," J. Phys. A: Math. Gen., vol. 39, no. 47, 14649, 2006.

[15] M. Holzäpfel, T. Baumgratz, M. Cramer and M. B. Plenio, "Scalable reconstruction of unitary processes and Hamiltonians," Phys. Rev. A vol. 91, no. 4, 042129, 2015.

[16] K. Rudinger and R. Joynt, "Compressed sensing for Hamiltonian reconstruction," Phys. Rev. A, vol. 92, no. 5, 052322, 2015.

[17] C. Di Franco, M. Paternostro and M. S. Kim, "Hamiltonian tomography in an access-limited setting without state initialization," Phys. Rev. Lett., vol. 102, no. 18, 187203, 2009.

[18] S. T. Wang, D. L. Deng and L. M. Duan, "Hamiltonian tomography for quantum many-body systems with arbitrary couplings," New J. Phys., vol. 17, no. 9, 093017, 2015.

[19] J. Zhang and M. Sarovar, "Quantum Hamiltonian identification from measurement time traces," Phys. Rev. Lett., vol. 113, no. 8, 080401, 2014.

[20] Y. Wang, D. Dong, I. R. Petersen and J. Zhang, "An approximate algorithm for quantum Hamiltonian identification, its complexity and error analysis," Proc. 20th IFAC World Congr., Toulouse, France, 9-14 July, 2017.

[21] A. Sone and P. Cappellaro, "Dimension estimability via single-probe measurement," arXiv preprint, quant-ph, arXiv: 1702.03280, 2017.

[22] L. Ljung, System Identification: Theory for the User, New Jersey, Prentice Hall, 1999. 